\title{
Histone demethylase retinoblastoma binding protein 2 regulates the expression of $\alpha$-smooth muscle actin and vimentin in cirrhotic livers
}

\author{
Q. Wang $^{1 *}$, L.X. Wang ${ }^{2 *}$, J.P. Zeng ${ }^{3}$, X.J. Liu ${ }^{1}$, X.M. Liang ${ }^{1}$ and Y.B. Zhou ${ }^{1}$ \\ ${ }^{1}$ Department of Microbiology, Key Laboratory for Experimental Teratology of the Chinese Ministry of Education, \\ School of Medicine, Shandong University, Jinan, China \\ ${ }^{2}$ Department of Pharmacology, School of Medicine, Shandong University, Jinan, China \\ ${ }^{3}$ Department of Biochemistry, School of Medicine, Shandong University, Jinan, China
}

\begin{abstract}
Liver cirrhosis is one of the most common diseases of Chinese patients. Herein, we report the high expression of a newly identified histone 3 lysine 4 demethylase, retinoblastoma binding protein 2 (RBP2), and its role in liver cirrhosis in humans. The siRNA knockdown of RBP2 expression in hepatic stellate cells (HSCs) reduced levels of $\alpha$-smooth muscle actin ( $\alpha$-SMA) and vimentin and decreased the proliferation of HSCs; and overexpression of RBP2 increased $\alpha$-SMA and vimentin levels. Treatment with transforming growth factor $\beta$ (TGF- $\beta$ ) upregulated the expression of RBP2, $\alpha-S M A$, and vimentin, and the siRNA knockdown of RBP2 expression attenuated TGF- $\beta$-mediated upregulation of $\alpha$-SMA and vimentin expression and HSC proliferation. Furthermore, RBP2 was highly expressed in cirrhotic rat livers. Therefore, RBP2 may participate in the pathogenesis of liver cirrhosis by regulating the expression of $\alpha$-SMA and vimentin. RBP2 may be a useful marker for the diagnosis and treatment of liver cirrhosis.
\end{abstract}

Key words: RBP2; Liver cirrhosis; Fibrosis; $\alpha$-SMA; Vimentin; HSC

\section{Introduction}

Liver cirrhosis, a chronic hepatic disease, is characterized by changes in the hepatic lobule structure and alterations in the vascular system of the liver. Liver fibrosis is the primary presentation of the disease, and hepatic stellate cells (HSCs) play a key role in fibrogenesis (1-3). When in a quiescent state, HSCs can store vitamin A, but they are activated by fibrogenic stimuli. The activation of HSCs is the predominant event in liver fibrosis. Stimuli such as transforming growth factor $\beta$ (TGF- $\beta$ ), tumor necrosis factor $\alpha$ (TNF- $\alpha$ ), and platelet-derived growth factor (PDGF) contribute to this process $(4,5)$. Activated HSCs can undergo transdifferentiation, which results in the formation of myofibroblasts that express the activation markers $\alpha$-smooth muscle actin ( $\alpha$-SMA) and vimentin. In addition, extracellular matrix synthesis and cell proliferation are enhanced. The expression of E-cadherin, which mediates the link between adjacent cells, is often decreased during fibrogenesis. Fibrosis can change the liver architecture and lead to dysfunction, ultimately resulting in liver cirrhosis.

Fibrosis is reversible, and when the pathogenic factors are eliminated, fibrotic structures can be absorbed gradually. Attenuating $\alpha-S M A$ and vimentin expression or extracellular matrix synthesis can block liver fibrosis (6). However, if fibrosis progresses, liver cirrhosis occurs, and the normal hepatic lobule architecture is disrupted, which can lead to liver organ failure and death. We have investigated the molecular mechanisms responsible for liver cirrhosis, but there is no consensus regarding the details.

Retinoblastoma binding protein 2 (RBP2), an important epigenetic molecule, has been implicated in cancer and other diseases $(7,8)$. This protein is a newly identified histone demethylase and is a member of the

Correspondence: Y.B. Zhou, Department of Microbiology, School of Medicine, Shandong University, Jinan 250012, China. Fax: +86531-8838-2502. E-mail: zyb@sdu.edu.cn

${ }^{*}$ These authors contributed equally to this study.

Received December 3, 2012. Accepted May 17, 2013. First published online September 16, 2013. 
Jumonji/AT-rich interactive domain (JARID) protein family. RBP2 has histone demethylase activity and controls the expression of multiple genes. It specifically targets tri- and di-methylated lysine 4 of histone 3 (H3-K4) for demethylation to regulate gene expression. The deregulation of RBP2 may lead to human diseases, especially developmental disorders (9-11).

We previously found that RBP2 is overexpressed in gastric cancer and that the inhibition of this demethylase could trigger the senescence of cancer cells (12). In addition, we found that RBP2 is overexpressed in hepatocellular carcinoma (data not shown); however, the role of this protein in liver cirrhosis, a condition that is closely associated with hepatocellular carcinoma, is unknown. In this study, we determined whether RBP2 contributes to the pathogenesis of liver cirrhosis in hepatic cells and in a cirrhotic rat model.

\section{Material and Methods}

\section{Clinical specimens}

We obtained 21 diseased and healthy liver samples from patients with liver cirrhosis treated in the Pathology Department of Bengbu Medical University, Anhui Province, China, from 2008 to 2009. The samples were collected immediately after surgery, stored in formalin, and then embedded in paraffin. None of the patients had received any treatment prior to surgery. Our study was approved by the local Ethics Committee.

\section{Immunohistochemistry}

Formalin-fixed, paraffin-embedded sections of liver tissue (5- $\mu \mathrm{m}$ thick) were deparaffinized and dehydrated with xylene and a graded series of alcohol. Antigen retrieval involved heat treatment in $0.1 \mathrm{M}$ citrate buffer at $\mathrm{pH}$ 6.0. Then, $3 \% \mathrm{H}_{2} \mathrm{O}_{2}$ was used to block the endogenous peroxidase activity, and the slides were incubated with goat serum for $30 \mathrm{~min}$ to eliminate non-specific antigens. The slides were then incubated with a monoclonal rabbit anti-human RBP2 antibody (Sigma, USA) overnight at $4^{\circ} \mathrm{C}$, followed by the appropriate secondary antibodies for $30 \mathrm{~min}$. Finally, antibody binding was detected using the avidin-biotin-peroxidase method with 3,3'-diaminobenzidine staining (Vector Laboratories, USA). The staining was analyzed under a microscope (Olympus BX60, Japan) equipped with a digital camera.

\section{Cell culture and siRNA interference}

LX-2 cells were grown in DMEM (Gibco, USA) supplemented with $12 \%$ fetal bovine serum (Gibco), $100 \mathrm{U} / \mathrm{mL}$ penicillin, and $100 \mu \mathrm{g} / \mathrm{mL}$ streptomycin. The cells were treated with $5 \mathrm{ng} / \mathrm{mL}$ TGF- $\beta$ (Peprotech, USA) for $48 \mathrm{~h}$, and then the cells were harvested for mRNA and protein analyses. The cells were cultured on 6 -well plates $\left(1.0 \times 10^{5}\right.$ cells/well) overnight and then transfected with Lipofectamine 2000 (Invitrogen, USA) before siRNA transfection. Chemically modified Stealth ${ }^{\mathrm{TM}}$ siRNA was obtained from Invitrogen. The sequences of the control and RBP2 siRNAs were 5'-CCU ACA UCC CGA UCG AUG AUG UUG-3' and 5'-CCA GCA CCA CCU CCU UCC UUC AUA A-3', respectively.

\section{RNA extraction, RT-PCR and real-time PCR}

Total RNA was extracted from cultured LX-2 cells using the Trizol reagent method (Invitrogen), and $3 \mu \mathrm{g}$ total RNA was reverse-transcribed using the Revert Aid First-Strand DNA Synthesis kit (Fermentas, Canada). The PCR products were analyzed by $1.5 \%$ agarose gel electrophoresis. The primers are listed in Table 1. The relative levels of mRNA were analyzed using SYBR green or Taqman probe real-time quantitative RT-PCR (Applied Biosystems, USA) and were normalized to the mRNA level of $\beta$-actin. The results were analyzed using the traditional $2^{-\Delta \Delta \mathrm{Ct}}$ method.

\section{Western blot analysis}

$\mathrm{LX}-2$ cells were lysed in lysis buffer $(50 \mathrm{mM}$ Tris- $\mathrm{HCl}$, $\mathrm{pH} 6.8,5 \%$ glycerol, $2 \%$ SDS, $1.55 \%$ DTT). The lysates were resolved by SDS-PAGE, and the bands were detected using enhanced chemiluminescence (Millipore, USA). The primary antibodies were a monoclonal rabbit anti-RBP2 antibody (Bethyl Laboratories, USA), a monoclonal rat anti-human vimentin antibody (both 1:1000; R\&D Systems, USA), a polyclonal rabbit anti-human $\alpha$ SMA antibody (1:500; BIOSS, China), and an anti- $\beta$-actin antibody (1:10,000; Sigma).

\section{Plasmid transfection and clonal formation}

LX-2 cell cultures were transfected with an RBP2 overexpression plasmid using the X-tremeGENE 9 DNA Transfection Reagent (Roche Applied Science, USA) for $48 \mathrm{~h}$. RBP2-overexpressing LX-2 cells and cells treated with RBP2 siRNA or TGF- $\beta$ were seeded on 6 -well plates (500 cells/well) and cultured for 2 weeks. The cells were then stained with Giemsa for $10 \mathrm{~min}$ after methanol fixation. The number of colonies of more than 50 cells was counted.

Table 1. PCR primers.

\begin{tabular}{ll}
\hline Gene & \multicolumn{1}{c}{ Primers } \\
\hline RBP2 & 5'-GCTGCTGCAGCCAAAGTTG-3' (forward) \\
& 5'-AGCATCTGCTAACTGGTC-3' (reverse) \\
$\alpha$-SMA & $5^{\prime}$-AGCAGGCCAAGGGGCTATATAA-3' (forward) \\
& $5^{\prime}$-TTCGTAGCTGTCTTTTTGTCCCA-3' (reverse) \\
Vimentin & $5^{\prime}$-AAAACACCCTGCAATCTTTCAGA-3' (forward) \\
& 5'-CACTTTGCGTTCAAGGTCAAGAC-3' (reverse) \\
$\beta$-actin & $5^{\prime}$-AGTTGCGTTACACCCTTTCTTG-3' (forward) \\
& $5^{\prime}$-CACCTTCACCGTTCCAGTTTT-3' (reverse) \\
\hline
\end{tabular}




\section{Cirrhosis model}

Wistar rats (males) were injected subcutaneously with

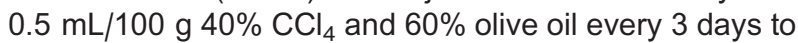
induce liver cirrhosis. The rats were also given water containing $10 \%$ alcohol and food with a high fat content and a low protein content. The weight of each rat was measured every 4 days. Six weeks later, the rats were killed, and their livers and spleens were harvested and analyzed. A portion of each liver was stored in formalin for sectioning and immunohistochemical staining, and the other portion was stored in liquid nitrogen.

\section{Statistical analysis}

Quantitative data are reported as means \pm SD or SE. The Student $t$-test was used to analyze the differences between groups. $\mathrm{P}<0.05$ was considered to be statistically significant.

\section{Results}

\section{RBP2 is overexpressed in cirrhotic human liver tissues}

RBP2 is deregulated in many diseases $(8,12)$. In this study, we found that RBP2 expression was greater in cirrhotic human livers than in normal livers (Figure 1). Because of this finding, we investigated the role that RBP2 plays in the initiation of liver cirrhosis and to identify the pathways involved.

\section{Blocking RBP2 expression reduced $\alpha$-SMA and} vimentin levels and inhibited HSC proliferation

Next, we analyzed the function of RBP2 in LX-2 HSCs, immortalized activated human HSCs, which we used to investigate the pathogenesis of liver fibrosis related to liver cirrhosis. siRNA knockdown reduced the RBP2 mRNA and protein levels (Figure $2 A$ and $B$ ), and the expression of $\alpha$-SMA and vimentin in LX-2 cells was
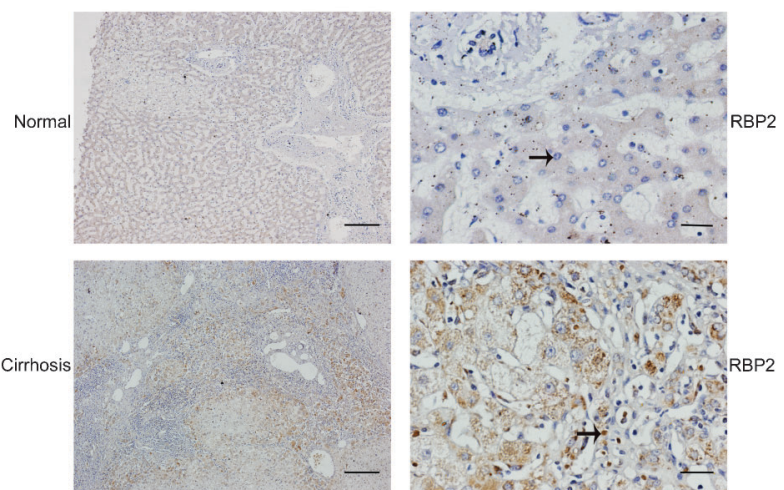

Figure 1. The expression of retinoblastoma binding protein 2 (RBP2) is upregulated in cirrhotic livers. The arrows show the negative (normal) and positive (cirrhosis) cells. Magnification bars $=40 \mu \mathrm{m}$ (left panels) and $10 \mu \mathrm{m}$ (right panels). suppressed by RBP2 depletion (Figure 2A and C). The suppression of RBP2 also inhibited the proliferation of LX-2 HSCs (Figure 2C). These results indicate that RBP2 regulates the expression of $\alpha$-SMA and vimentin and affects the proliferation of $L X-2$ cells.

\section{RBP2 overexpression upregulated $\alpha$-SMA and} vimentin expression in HSCs, similar to the results of TGF- $\beta$ treatment

To verify the regulation of $\alpha$-SMA and vimentin expression by RBP2, we transfected an RBP2 overexpression plasmid into LX-2 HSC cells. The $\alpha$-SMA and vimentin expression levels were markedly increased by RBP2 overexpression. HSCs can be activated by TGF- $\beta$, TNF- $\alpha$, and PDGF $(4,5)$, and thus we treated $L X-2$ cells with TGF- $\beta$ ( $5 \mathrm{ng} / \mathrm{mL})$ for $48 \mathrm{~h}$ and examined the $\alpha$-SMA and vimentin expression levels. The expression levels of both factors were upregulated, as was the expression of RBP2 (Figure 2A-C). RBP2 overexpression had effects on HSCs similar to those mediated by TGF- $\beta$ treatment. This result together with the fact that RBP2 depletion downregulated $\alpha$-SMA and vimentin expression led us to hypothesize that the upregulation of $\alpha$-SMA and vimentin expression by TGF- $\beta$ may be mediated by RBP 2 .

\section{Inhibition of RBP2 expression attenuated} upregulation of $\alpha$-SMA and vimentin expression and HSC proliferation mediated by TGF- $\beta$

When we pretreated LX-2 cells with RBP2 siRNA to suppress RBP2 expression, the TGF- $\beta$-upregulated expression of $\alpha$-SMA and vimentin was suppressed (Figure $3 \mathrm{~A}$ and $\mathrm{B}$ ). The siRNA knockdown of RBP2 also attenuated proliferation of LX-2 cells induced by TGF- $\beta$ (Figure $3 \mathrm{C}$ ). These results indicate that RBP2 participates in, and is required for, fibrosis-associated signaling mediated by TGF- $\beta$ in HSCs, suggesting that RBP2 plays an important role in the pathogenesis of liver cirrhosis.

\section{Role of RBP2 regulation in the cirrhotic rat mode}

To confirm the role of RBP2 regulation in vivo, we used the cirrhotic rat model. We performed hematoxylin and eosin staining of rat livers (Figure 4A) and assessed each rat's weight, liver index (liver weight/rat weight), and spleen index (spleen weight/rat weight; see Supplementary Figure S1). RBP2 was overexpressed in cirrhotic rat livers (Figure 4B and C). These results further indicate that the deregulation of RBP2 plays a role in liver cirrhosis, and the effects of RBP2 deregulation may be important in the pathogenesis of this condition.

\section{Discussion}

Liver cirrhosis is one of the most common diseases in Chinese patients. In this study, we found that a newly identified H3-K4 demethylase, RBP2, is highly expressed in cirrhotic human tissues. The siRNA knockdown of 
A

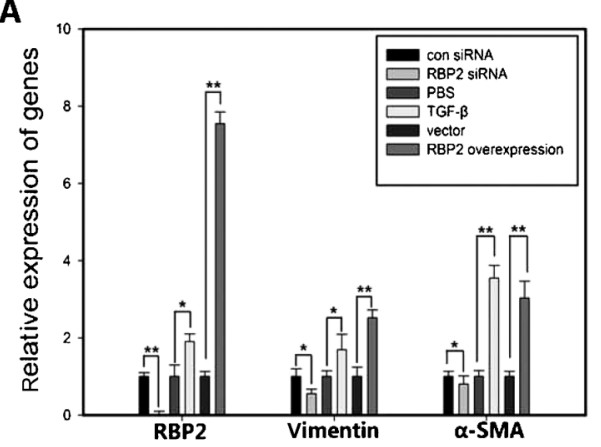

C

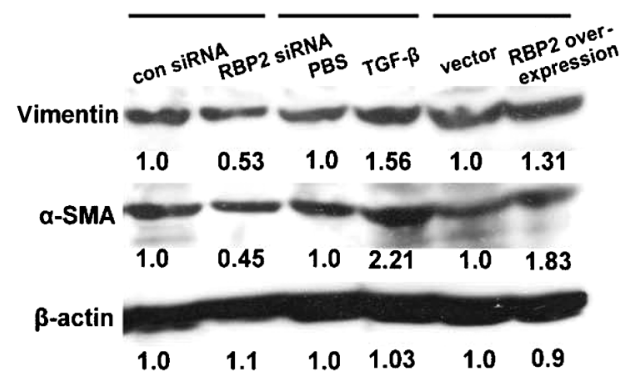

B

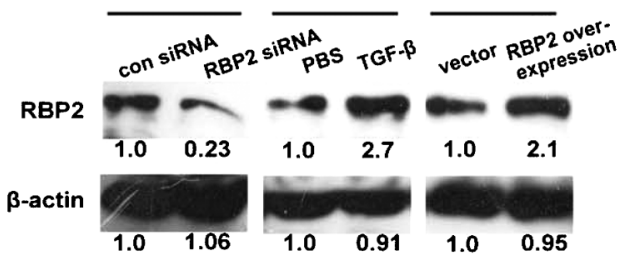

D
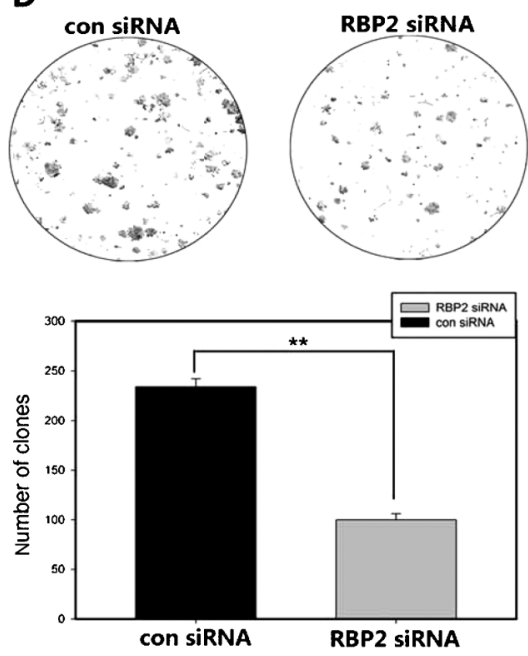

Figure 2. The $\alpha$-SMA and vimentin expression levels are regulated by RBP2 in LX- 2 cells, and the expression of RBP2 is upregulated by transforming growth factor $\beta$ (TGF- $\beta$ ). A, RT-PCR analysis revealed that the mRNA levels of RBP2, $\alpha$-SMA and vimentin were upregulated by TGF- $\beta(5 \mathrm{ng} / \mathrm{mL})$ and RBP2 overexpression and were downregulated by the suppression of RBP2 expression using siRNA. Data are reported as means $\pm \mathrm{SD}$ from 3 experiments (Student $t$-test; ${ }^{*} \mathrm{P}<0.05,{ }^{*} \mathrm{P}<0.01$ ). $B$, Western blot analysis of the protein levels of RBP2 with RBP2 siRNA, TGF- $\beta$ and RBP2 overexpression plasmid treatment. Data are representative of 3 experiments. $C$, Western blot analysis of the protein levels of $\alpha$-SMA and vimentin with RBP2 siRNA, TGF- $\beta$ and RBP2 overexpression plasmid treatment. Data are representative of 3 experiments. $D$, Proliferation of LX-2 cells when treated with RBP2 siRNA. The Student $t$-test was used for analysis $\left({ }^{* *} \mathrm{P}<0.01\right)$.

RBP2 expression in HSCs suppressed $\alpha$-SMA and vimentin expression and decreased the proliferation of HSCs. Overexpression of RBP2 enhanced the expression of $\alpha$-SMA and vimentin. TGF- $\beta$ treatment upregulated RBP2, $\alpha-S M A$, and vimentin levels and increased HSC proliferation. In addition, RBP2 siRNA knockdown attenuated the upregulation of $\alpha$-SMA and vimentin expression and HSC proliferation. RBP2 was highly expressed in cirrhotic rat livers. RBP2 may participate in the pathogenesis of liver cirrhosis by regulating $\alpha$-SMA and vimentin expression and may be a useful marker for the diagnosis and treatment of liver cirrhosis.

Liver disease is a major threat to the health of people all over the world. Liver fibrosis is a precursor of many serious liver diseases including liver cirrhosis, and should be addressed. HSCs are of key importance in liver fibrosis; they are quiescent in healthy livers, and their proliferation level is low. They can store vitamin A. In response to some stimuli, HSCs can be activated and become more contractile (13). The activation and proliferation of HSCs result in the increased production of extracellular matrix, which facilitates the re-construction of liver tubules. Activated HSCs can transdifferentiate into myofibroblasts that express increased levels of $\alpha$-SMA and vimentin. Liver fibrosis may be coupled with the loss of E-cadherin, a protein associated with cell-cell junctions. E-cadherin, an important epithelial marker (14), is closely associated with the epithelial-mesenchymal transition, which enhances the invasiveness and metastatic ability of malignant transformed cells. Some factors, especially TGF- $\beta$, participate in liver fibrosis $(15,16)$. TGF- $\beta$ can induce the expression of markers of HSC activation, and 

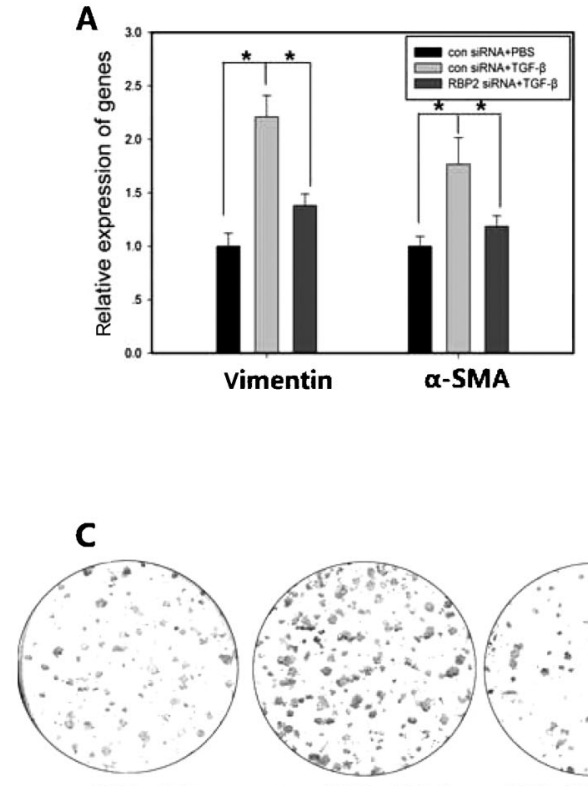

con siRNA+PBS

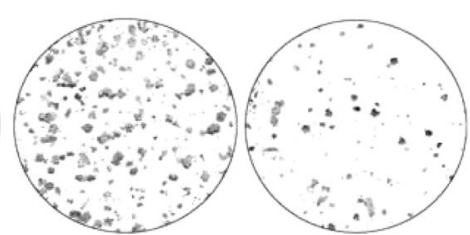

con SIRNA+TGF- $\beta$

RBP2 SIRNA + TGF- $\beta$
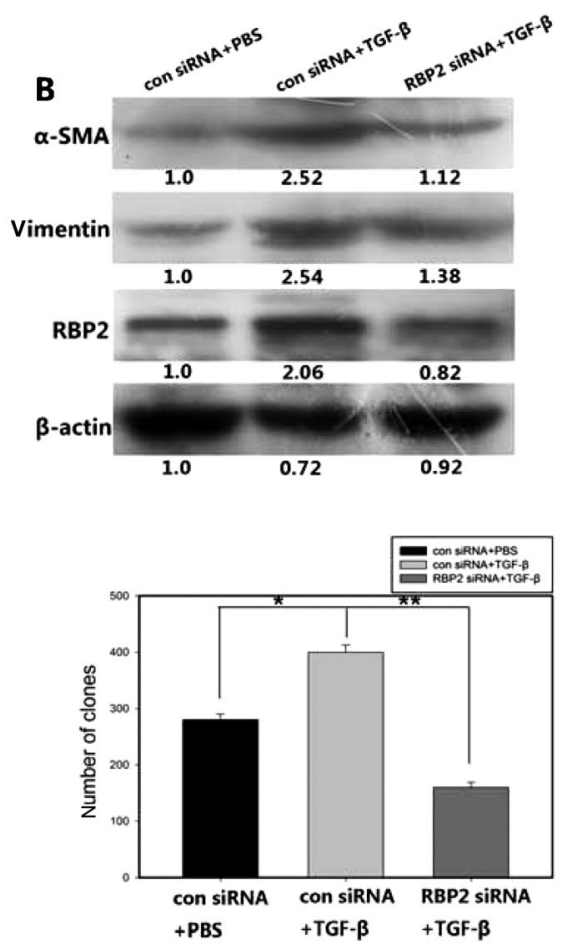

Figure 3. The activation of LX-2 cells by TGF- $\beta$ was attenuated by RBP2 suppression. Quantification $(A)$ and Western blot analysis $(B)$ of $\alpha$-SMA and vimentin after treatment with RBP2 siRNA and TGF- $\beta(5 \mathrm{ng} / \mathrm{mL})$. Data are reported as means \pm SD from 3 experiments (Student $t$-test; ${ }^{*} \mathrm{P}<0.05$ ). $C$, Proliferation of LX-2 cells treated with RBP2 siRNA and TGF- $\beta$. The Student $t$-test was used for analysis $\left({ }^{*} \mathrm{P}<0.05,{ }^{* *} \mathrm{P}<0.01\right)$.

any factor that stimulates TGF- $\beta$ signaling can lead to HSC activation $(17,18)$. Liver fibrosis resulting from the activation of HSCs can progress to liver cirrhosis if the lesions cannot heal. Liver cirrhosis substantially impairs liver function and has many serious presentations and complications. However, the detailed mechanisms of the activation of HSCs and of the conversion from liver fibrosis to cirrhosis have not been fully elucidated.

RBP2, a recently identified H3-K4 demethylase, participates in the pathogenesis of many types of diseases, especially cancer, by epigenetically regulating specific genes. This protein belongs to the JARID demethylase protein family $(19,20)$. RBP2 has histone demethylase activity and demethylates $\mathrm{H} 3-\mathrm{K} 4$ me3 and me2. This protein was first investigated in the context of development, and we have reported its deregulation in gastric cancer (12) and hepatocellular carcinoma (data not shown). In cancerous tissues, RBP2 can promote cell proliferation and restrict cell senescence by directly regulating cyclin-dependent kinase inhibitors (12). Recently, the upregulation of RBP2/KDM5A expression was found to be a characteristic of drug-tolerant cancer cell subpopulations (8), namely cancer stem cells, highlighting the role of RBP2 in cancer. However, there have been few investigations of the function and regulation of
RBP2 in other types of diseases. In this study, we first analyzed the expression of RBP2 and found that it is highly expressed in cirrhotic liver tissue, a result that suggests that RBP2 may play a role in the pathogenesis of this disease. In addition, liver cirrhosis may be associated with epigenetic regulation in addition to the gene mutations reported by other investigators $(21,22)$.

The expression of the mesenchymal marker vimentin is upregulated when HSCs are activated. Another activation marker, $\alpha-S M A$, is also upregulated in the early stages of HSC activation. Together with the expression of $\alpha$-SMA and vimentin, the expression of RBP2 was found to be upregulated in activated HSCs. Furthermore, the siRNA knockdown of RBP2 expression downregulated $\alpha-S M A$ and vimentin expression, and RBP2 overexpression enhanced the expression of these two markers. Thus, RBP2 may participate in the activation of HSCs by regulating $\alpha-S M A$ and vimentin expression. Of key importance is the result that blocking RBP2 expression could attenuate the upregulation of $\alpha$-SMA and vimentin expression mediated by TGF- $\beta$. In addition, the proliferation of HSCs induced by TGF- $\beta$ was attenuated by the suppression of RBP2 expression. These results indicate that RBP2 is involved in HSC activation and may participate in the pathogenesis of liver fibrosis. 
A
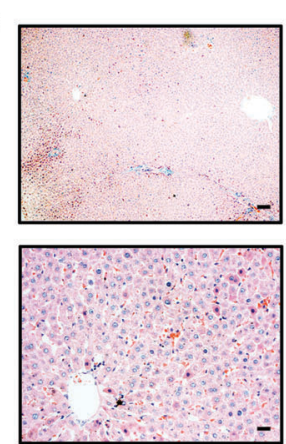

Normal
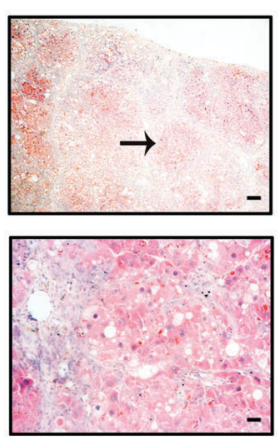

Cirrhosis
B

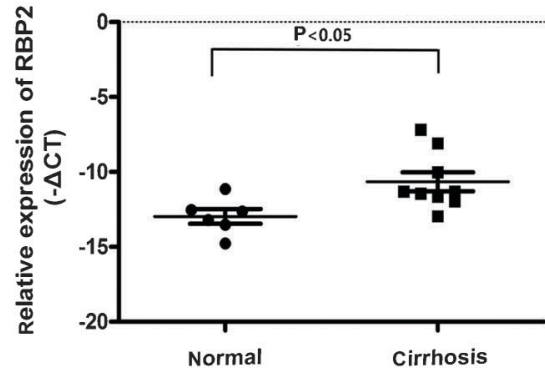

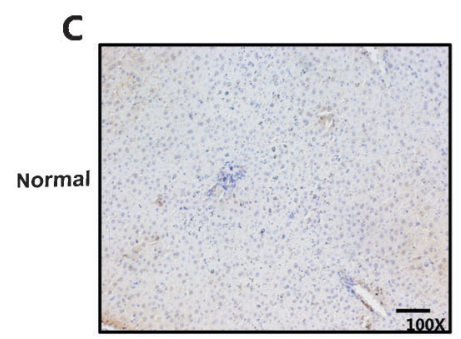
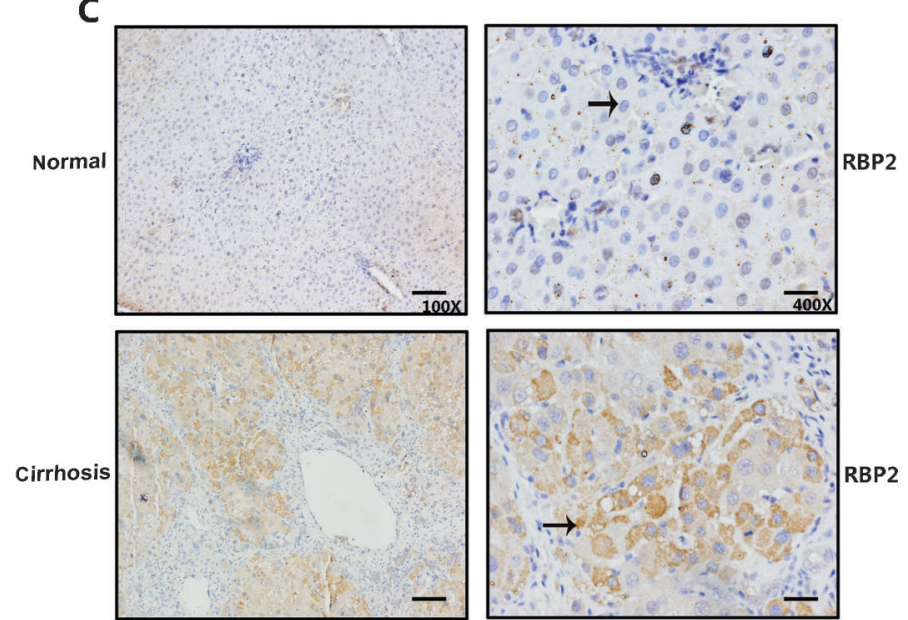

Figure 4. Upregulation of RBP2 in cirrhotic rat livers. A, Hematoxylin and eosin staining of normal and cirrhotic livers. The arrow shows the formed sclerotic nodules in liver. Magnification bar $=10 \mu \mathrm{m} . B, \mathrm{RBP} 2 \mathrm{mRNA}$ expression in normal and cirrhotic livers. Each dot represents 1 rat. The Student $t$-test was used for analysis. $C$, RBP2 expression in normal and cirrhotic livers. The arrows show the negative (normal) and positive (cirrhosis) cells. Magnification bars $=40 \mu \mathrm{m}$ (left panels) and $10 \mu \mathrm{m}$ (right panels).

In HSCs, the expression of $\alpha$-SMA and vimentin was positively regulated by RBP2, but determining whether the expression of $\alpha$-SMA and vimentin is directly regulated by RBP2 requires further investigation. E-cadherin antagonizes TGF- $\beta 1$ gene induction in HSCs by inhibiting RhoA-dependent Smad3 phosphorylation (14), and the expression of $\alpha$-SMA and vimentin was previously found to be positively regulated by TGF- $\beta 1$ signaling $(23,24)$. The overexpression of RBP2 may inhibit E-cadherin expression by promoting the binding of RBP2 to the CCGCCC DNA motif in the E-cadherin promoter through RBP2's DNA binding domain. This inhibition of E-cadherin expression would attenuate the antagonization of TGF- $\beta 1$-activated genes ( $\alpha$-SMA and vimentin), thus leading to the upregulation of $\alpha$-SMA and vimentin expression in cirrhotic livers. This upregulation would contribute to the pathogenesis of liver fibrosis, but further investigation is required. Our experiments confirmed that RBP2 plays a role in the pathogenesis of liver fibrosis and liver cirrhosis, which may be an important epigenetic regulation of this disease. High RBP2 expression may be a diagnostic marker for liver cirrhosis, and suppressing RBP2 expression may be useful for the treatment of this condition.

\section{Supplementary Material}

Click here to view [pdf].

\section{Acknowledgments}

We thank the National Natural Science Foundation of China (Grants \#81001098, \#81171536, \#81170514, \#81172354 and \#81272654), the National Basic Research Program of China (Grant of the 973 Program \#2012CB911202), and the Science Foundation of Shandong Province (Grants \#ZR2009CZ001, \#ZR2009CM002 and \#ZR2010HZ003). 


\section{References}

1. Moreira RK. Hepatic stellate cells and liver fibrosis. Arch Pathol Lab Med 2007; 131: 1728-1734.

2. Calvaruso V, Maimone S, Gatt A, Tuddenham E, Thursz M, Pinzani $M$, et al. Coagulation and fibrosis in chronic liver disease. Gut 2008; 57: 1722-1727, doi: 10.1136/gut.2008. 150748.

3. Kisseleva T, Brenner DA. Hepatic stellate cells and the reversal of fibrosis. J Gastroenterol Hepatol 2006; 21 (Suppl 3): S84-S87, doi: 10.1111/j.1440-1746.2006.04584.x.

4. Narmada BC, Chia SM, Tucker-Kellogg L, Yu H. HGF regulates the activation of TGF-beta1 in rat hepatocytes and hepatic stellate cells. J Cell Physiol 2013; 228: 393-401, doi: $10.1002 / j \mathrm{jcp} .24143$

5. Connolly MK, Bedrosian AS, Mallen-St CJ, Mitchell AP, Ibrahim J, Stroud A, et al. In liver fibrosis, dendritic cells govern hepatic inflammation in mice via TNF-alpha. J Clin Invest 2009; 119: 3213-3225.

6. Chen JY, Chen HL, Cheng JC, Lin HJ, Tung YT, Lin CF, et al. A Chinese herbal medicine, Gexia-Zhuyu Tang (GZT), prevents dimethylnitrosamine-induced liver fibrosis through inhibition of hepatic stellate cells proliferation. $J$ Ethnopharmacol 2012; 142: 811-818, doi: 10.1016/j.jep. 2012.06.005.

7. Benevolenskaya EV, Murray $\mathrm{HL}$, Branton $\mathrm{P}$, Young RA, Kaelin WG Jr. Binding of $p R B$ to the PHD protein RBP2 promotes cellular differentiation. Mol Cell 2005; 18: 623635, doi: 10.1016/j.molcel.2005.05.012.

8. Sharma SV, Lee DY, Li B, Quinlan MP, Takahashi F, Maheswaran S, et al. A chromatin-mediated reversible drug-tolerant state in cancer cell subpopulations. Cell 2010; 141: 69-80, doi: 10.1016/j.cell.2010.02.027.

9. Christensen J, Agger K, Cloos PA, Pasini D, Rose S, Sennels $L$, et al. RBP2 belongs to a family of demethylases, specific for tri- and dimethylated lysine 4 on histone 3 . Cell 2007; 128: 1063-1076, doi: 10.1016/j.cell.2007.02.003

10. Klose RJ, Yan Q, Tothova Z, Yamane K, ErdjumentBromage $\mathrm{H}$, Tempst $\mathrm{P}$, et al. The retinoblastoma binding protein RBP2 is an H3K4 demethylase. Cell 2007; 128: 889900, doi: 10.1016/j.cell.2007.02.013

11. Lopez-Bigas N, Kisiel TA, Dewaal DC, Holmes KB, Volkert TL, Gupta S, et al. Genome-wide analysis of the H3K4 histone demethylase RBP2 reveals a transcriptional program controlling differentiation. Mol Cell 2008; 31: 520-530, doi: 10.1016/j.molcel.2008.08.004.

12. Zeng J, Ge Z, Wang L, Li Q, Wang N, Bjorkholm M, et al. The histone demethylase RBP2 is overexpressed in gastric cancer and its inhibition triggers senescence of cancer cells. Gastroenterology 2010; 138: 981-992, doi: 10.1053/j.gastro.2009.10.004.

13. Muhanna N, Doron S, Wald O, Horani A, Eid A, Pappo O, et al. Activation of hepatic stellate cells after phagocytosis of lymphocytes: A novel pathway of fibrogenesis. Hepatology 2008; 48: 963-977, doi: 10.1002/hep.22413.
14. Cho IJ, Kim YW, Han CY, Kim EH, Anderson RA, Lee YS et al. E-cadherin antagonizes transforming growth factor beta1 gene induction in hepatic stellate cells by inhibiting RhoA-dependent Smad3 phosphorylation. Hepatology 2010; 52: 2053-2064, doi: 10.1002/hep.23931.

15. Yang KL, Chang WT, Chuang CC, Hung KC, Li El. Antagonizing TGF-beta induced liver fibrosis by a retinoic acid derivative through regulation of ROS and calcium influx. Biochem Biophys Res Commun 2008; 365: 484-489, doi: 10.1016/j.bbrc.2007.10.203.

16. Dooley S, Hamzavi J, Ciuclan L, Godoy P, Ilkavets I, Ehnert $\mathrm{S}$, et al. Hepatocyte-specific Smad7 expression attenuates TGF-beta-mediated fibrogenesis and protects against liver damage. Gastroenterology 2008; 135: 642-659, doi: 10.1053/j.gastro.2008.04.038.

17. Shimada H, Staten NR, Rajagopalan LE. TGF-beta1 mediated activation of Rho kinase induces TGF-beta2 and endothelin-1 expression in human hepatic stellate cells. $J$ Hepatol 2011; 54: 521-528, doi: 10.1016/j.jhep.2010.07.026.

18. Liu Y, Wen XM, Lui EL, Friedman SL, Cui W, Ho NP, et al. Therapeutic targeting of the PDGF and TGF-beta-signaling pathways in hepatic stellate cells by PTK787/ZK22258. Lab Invest 2009; 89: 1152-1160, doi: 10.1038/labinvest.2009.77.

19. Roesch A, Fukunaga-Kalabis M, Schmidt EC, Zabierowski SE, Brafford PA, Vultur A, et al. A temporarily distinct subpopulation of slow-cycling melanoma cells is required for continuous tumor growth. Cell 2010; 141: 583-594, doi: 10.1016/j.cell.2010.04.020

20. Tabernero MD, Espinosa AB, Maillo A, Rebelo O, Vera JF, Sayagues JM, et al. Patient gender is associated with distinct patterns of chromosomal abnormalities and sex chromosome linked gene-expression profiles in meningiomas. Oncologist 2007; 12: 1225-1236, doi: 10.1634/theoncologist.12-101225.

21. Mitchell MM, Lleo A, Zammataro L, Mayo MJ, Invernizzi P, Bach $N$, et al. Epigenetic investigation of variably $X$ chromosome inactivated genes in monozygotic female twins discordant for primary biliary cirrhosis. Epigenetics 2011; 6: 95-102, doi: 10.4161/epi.6.1.13405.

22. Mann J, Chu DC, Maxwell A, Oakley F, Zhu NL, Tsukamoto $\mathrm{H}$, et al. MeCP2 controls an epigenetic pathway that promotes myofibroblast transdifferentiation and fibrosis. Gastroenterology 2010; 138: 705-714, doi: 10.1053/j.gastro. 2009.10.002.

23. Satish L, Gallo PH, Baratz ME, Johnson S, Kathju S. Reversal of TGF-beta1 stimulation of alpha-smooth muscle actin and extracellular matrix components by cyclic AMP in Dupuytren's-derived fibroblasts. BMC Musculoskelet Disord 2011; 12: 113, doi: 10.1186/1471-2474-12-113.

24. Nishida $Y$, Shibata K, Yamasaki M, Sato $Y$, Abe M. A possible role of vimentin on the cell surface for the activation of latent transforming growth factor-beta. FEBS Lett 2009; 583: 308-312, doi: 10.1016/j.febslet.2008.12.051. 